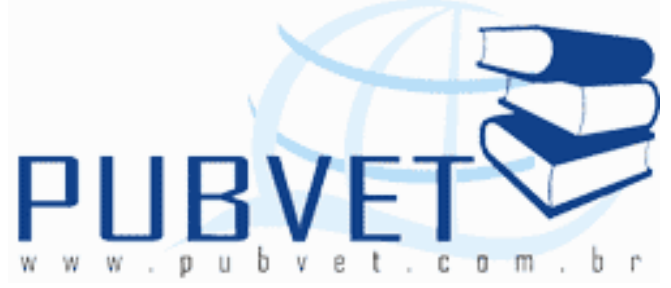

PUBVET, Publicações em Medicina Veterinária e Zootecnia.

\title{
Simulação de dados para avaliação econômica do sistema de cria de bovinos de corte em pastagem de Brachiaria brizantha cv. Marandu
}

Girlei Gonçalves da Costa $^{1}$; Rodrigo Zaiden Taveira ${ }^{2}$; Osvaldo José da Silveira Neto $^{2}$; Vanderly Alves de Brito Júnior ${ }^{1}$

${ }^{1}$ Acadêmico do curso de Zootecnia - UEG - UnU - São Luís de Montes Belos ${ }^{2}$ Docente e Pesquisador - Curso de Zootecnia - UEG - UnU - São Luís de Montes Belos

\section{Resumo}

O correto entendimento e funcionamento de todas as etapas envolvidas no processo de produção de bezerros, leva ao produtor a tomadas de decisão quanto a implantar uma nova tecnologia. Tendo em vista essa necessidade objetivou-se com este trabalho demonstrar os resultados através de simulação de produção, com um rebanho base de 500 fêmeas acima de 36 meses, submetidas à IATF, e 20 touros de repasse. Sendo simulados 5 (cinco) cenários diferentes: C1 60\%, C2 70\%, C3 80\%, C4 85\% e C5 90\% de natalidade. Constatou-se que índices de natalidade de $60 \%$ até $80 \%$ com descarte de $50 \%$ de fêmeas falhas, a Margem liquida fica negativa, ficando positiva apenas com índices acima de $80 \%$. O que pode ser visto nos cenários C4 e C5 onde se tem lucro de $R \$ 272.290,50$ e $R \$ 353.166,7 /$ ano.

Palavras-chave: tecnologia, fêmea, precocidade, produção, lucratividade. 


\title{
Data simulatiion for economical evaluation of beef cattle breed system in Brachiaria brizantha cv. Marandu pasture
}

\begin{abstract}
The correct knowledge of the function of all the phases involved in the process of calves production, helps the rural producer in the choice of news technologies that would be implanted. Considering this, the aim of this paper was to show results by the simulated data of production, considering the base livestock of female of 500 female over 36 months submitted to artificial insemination in fixed time and 20 bulls for posterior mate. It was simulated 05 different scenario considering increasing indexes of livestock birth, being: S1: $60 \%$, S2: $70 \%$, S3: $80 \%$, S4: $85 \%$ e S5: $90 \%$ of birth. Can be verify that birth indexes ranging from $60 \%$ to $80 \%$, sold $50 \%$ of hole female, showing negative liquid margin, become positive with indexes over $80 \%$, that could observed in the S4 and S5, whose showing earnings of $R \$ 272,290.50$ and $R \$$ $353,166.70 /$ year, respectively. The increase in the birth rate is one of the most way that increase the economic efficiency of systems of calves production.
\end{abstract}

Keywords: earning, female, precocity, production, technology.

\section{Introdução}

Sabe-se que a busca pela eficiência na produção de bovinos de corte, passa pelo correto entendimento e funcionamento de todas as etapas envolvidas no processo, desde a produção de insumos até a satisfação do consumidor. Nesse sentido, a fase de cria exerce importante impacto em todo o sistema, já que apresenta reflexos diretos na taxa de desfrute do rebanho, merecendo, portanto, atenção especial quanto aos seus custos, especialmente no valor gasto para se obter um bezerro por ciclo.

Os indicadores que fundamentam a eficiência do processo produtivo na fase de cria devem ser constantemente avaliados, dentre eles, conforme 
Lobato, (2001), destacam-se: taxa de prenhez (TP), taxa de natalidade (TN), taxa de desmama (TD), peso à desmama (PD), produtividade em quilos de bezerros desmamados/vaca exposta à reprodução e a eficiência por área destinada à cria (EA), os quais apresentam-se, em média, insatisfatórios e são ainda pouco estudados nos sistemas de produção de bovinos de corte.

Do ponto de vista econômico, esses índices precisam ser coletados e interpretados de maneira consciente e prática, evidenciando os pontos de gargalo da fase de cria. Percebe-se, no entanto, dificuldade por parte dos pecuaristas, em transformar esses índices em informações aplicáveis ao rebanho de cria, o que os coloca à margem do real custo de produção do bezerro.

Porém, o intervalo de gerações em bovinos de corte é longo para se avaliar resposta à seleção sobre uma única característica, devendo ser analisada e pautada no tempo (Meirelles, 2005).

Nesse sentido, a modelagem e a simulação de sistemas têm sido propostas como instrumentos da pesquisa que permitem realizar este tipo de estudo, minimizando custos e reduzindo o tempo de avaliação, observando os resultados econômicos em função das biotecnologias que são implantadas nos rebanhos, verificando custos e avaliando os resultados (Beretta, et. al., 2001).

Tendo em vista o exposto, objetivou-se simular em 05 diferentes situações a produção de bezerros num rebanho base de 500 matrizes da raça Nelore, com idade acima de 36 meses, sendo submetidas a inseminação artificial em tempo fixo (IATF) com 20 touros, durante os anos de 2012 a 2017.

\section{Material e Métodos}

No presente estudo foi realizada uma simulação da eficiência produtiva para a fase de cria na bovinocultura de corte, utilizando-se planilha do Microsoft Excel, 2003. 
Foi simulada a produção de bezerros num rebanho base de 500 matrizes da raça Nelore, com idade acima de 36 meses, sendo submetidas a inseminação artificial em tempo fixo (IATF) com 20 touros, durante os anos de 2012 a 2017. Foram realizadas cinco simulações distintas, sendo: C1: 60\% de taxa de natalidade; $50 \%$ de descarte de fêmeas vazias; primeiro serviço dos 24 aos 27 meses com taxa de natalidade de 50\%; C2: 70\% de taxa de natalidade; $50 \%$ de descarte de fêmeas vazias; primeiro serviço dos 21 aos 24 meses com taxa de natalidade de 50\%; C3: $80 \%$ de taxa de natalidade; 50\% de descarte de fêmeas vazias; primeiro serviço dos 21 aos 24 meses com taxa de natalidade de $60 \%$ e; C4: $85 \%$ de taxa de natalidade; $50 \%$ de descarte de fêmeas vazias; primeiro serviço dos 18 aos 21 meses com taxa de natalidade de 65\%; C5: 95\% de taxa de natalidade; 50\% de descarte de fêmeas vazias; primeiro serviço dos 18 aos 21 meses com taxa de natalidade de $70 \%$.

A propriedade possuía área de 600 ha de pastagem disponível, com taxa de lotação igual a 15 @/ha, considerando o ano inicial 2012 como o ano de introdução dos animais. Os custos de produção considerados foram: Mão-deobra: Manter 2 funcionários fixos. Operacional: recuperação de cercas e reparos na propriedade; Alimentação: manutenção da pastagem, suplementação mineral e proteinado; Sanidade composta por: vacinação obrigatória (Febre aftosa, Brucelose e Raiva) e preventiva (Vermífugo, Antiparasitário feito 2 vezes no ano e Carbúnculo sintomático); Reprodutivo: Protocolo de IATF e capital investido (sendo $6 \%$ do valor investido ao ano).

A renda da propriedade foi considerada como sendo da venda de bezerros e vacas descarte (vazia ou excedente). Foi avaliado o sistema durante 5 (cinco) anos contados a partir da data de introdução.

O resultado (lucro ou prejuízo) do sistema foi estimado utilizando-se o custo para produção dos bezerros e valor de venda dos animais de descarte, excedentes e desmamados.

As despesas consideradas foram relativas a todos os gastos com a produção e manutenção da propriedade; A Receita Bruta foi estimada como sendo todos os valores que entraram na propriedade oriunda das vendas de 
animais; $A$ Receita liquida foi dada pela fórmula $R L=R B-D$, onde $R L=$ receita liquida $\mathrm{RB}=$ receita bruta, $\mathrm{D}=$ despesa. $\mathrm{O}$ Saldo acumulado ano anterior foi dado pela fórmula $\mathrm{SA}=$ RLano atual $-\mathrm{SA}$ ano anterior; A Diferença de inventario foi dada por $\mathrm{DI}=\mathrm{I}-\mathrm{IAt}$, onde $\mathrm{DI}=$ diferença de inventario, $\mathrm{I}=$ inventario atual, $\mathrm{IAt}=$ inventario anterior; $(\mathrm{ML}=\mathrm{SA}+\mathrm{DI})$ onde $\mathrm{ML}=$ margem liquida, $\mathrm{SA}=$ saldo acumulado ano anterior, $\mathrm{DF}=$ diferença de inventario; $(\mathrm{Kg}$ $\mathrm{bd} / \mathrm{ha} / \mathrm{ano}=\mathrm{n}^{\circ}$ de animais/ $\mathrm{kg}$ de bezerros desmamado no ano); ( $\mathrm{Kg}$ $\mathrm{bd} / \mathrm{vaca} / \mathrm{ano}=\mathrm{kg}$ de bezerros desmamados ano $\left(\mathrm{n}^{\circ}\right.$ de vacas expostas); (Eficiência do sistema $=\mathrm{Kg}$ bd ano $/ 100 \mathrm{~kg}$ de vacas expostas)

A tabela 1 dada abaixo apresenta o custo de produção por ano válido para todos os sistemas considerados.

Tabela 1. Custo de produção por ano válido para todos os sistemas considerados.

\section{Item}


As receitas foram calculadas através da venda de vacas de descarte ao final da estação de monta e $100 \%$ dos bezerros machos aos 8 meses ou seja a desmama, além dos touros de reposição, o que pode ser observado na tabela 2

Tabela 2. Receitas ( $\mathrm{R} \$ /$ cabeça e $\mathrm{R} \$ / @$ ) por venda de diferentes categorias de animais, considerando o ciclo de um ano de produção.

\begin{tabular}{lcc}
\hline Categoria & \multicolumn{2}{c}{ Receitas } \\
\hline & $\mathbf{R} \mathbf{\$} /$ cabeça & $\mathbf{R} \mathbf{\$} @$ \\
\cline { 2 - 2 } Vaca de descarte & 80 \\
Touro & 80 \\
Macho de 0 a 8 meses & 600 & \\
\hline
\end{tabular}

\section{Resultados e Discussão}

A tabela 3 dada abaixo apresenta a composição do rebanho em função dos cenários avaliados considerando as diversas categorias estudadas.

Tabela 3. Composição do rebanho em função dos cenários avaliados considerando as categorias de fêmeas paridas, fêmeas de reposição, fêmeas vazias e touros.

Composição do rebanho

\begin{tabular}{lccccc}
\hline Cenário & C1 & C2 & C3 & C4 & C5 \\
\cline { 2 - 6 } Fêmeas paridas & $41 \%$ & $44 \%$ & $46 \%$ & $47 \%$ & $46 \%$ \\
Fêmeas de reposição & $45 \%$ & $44 \%$ & $47 \%$ & $49 \%$ & $51 \%$ \\
Fêmeas vazias & $11 \%$ & $9 \%$ & $4 \%$ & $1 \%$ & $0 \%$ \\
Touros & $3 \%$ & $3 \%$ & $3 \%$ & $3 \%$ & $3 \%$ \\
\hline
\end{tabular}


Tendo em vista as fêmeas paridas registra-se acréscimo de $5 \%$ do primeiro (C1) para o último cenário (C5), refletindo na maior quantidade de bezerros nascidos no sistema o que leva a um acréscimo na receita bruta do sistema, assim como reduz os gastos com animais ociosos na propriedade sendo refletido no numero de fêmeas de reposição.

Aumentando a taxa de natalidade de $60 \%$ no primeiro cenário avaliado (C1) para 90\% no cenário (C5) pode ser registrado aumento de $06 \%$ na quantidade de fêmeas irão compor o futuro rebanho de matrizes com maior valor genético, passando de $45 \%$ a $51 \%$. O aumento desse índice é de extrema importância dentro dos sistemas produtores de bovinos, já que reflete inclusive, na taxa de desfrute do rebanho.

Tendo em vista a avaliação das fêmeas vazias, percebe-se comportamento inverso, o qual decresceu gradativamente de acordo com o aumento da taxa de natalidade, chegando à zero. Comparando os cenários, nota se que partindo das vendas de $50 \%$ de vacas falhas no cenário (C1) até $100 \%$ no cenário (C5) considerado o cenário ideal, com descarte de todas as vacas falhas ao final da estação de monta. Esse procedimento ajudará a liberar áreas de pastagens para a melhor alimentação das fêmeas de reposição e vacas em produção aumentando o peso dos bezerros e proporcionando maior ganho de peso diário das bezerras que chegaram mais cedo à idade à puberdade, permitindo assim que as mesmas expressem todo seu potencial genético para precocidade sexual.

Corroborando com esses resultados, Beretta (2001) registrou novilhas de reposição na proporção de $53,1 \%$ do rebanho, tendo aumentado a taxa de natalidade pelo maior numero de bezerros nascidos, além de ter havido redução de animais ociosos chegando a zero o numero de vacas falhas com taxa de natalidade de $90 \%$.

A tabela 4 dada abaixo apresenta os resultados financeiros e de produção ( $\mathrm{kg}$ de bezerro desmamado/ha/ano,e $\mathrm{kg}$ de bezerro desmamado/ $100 \mathrm{Kg}$ de vaca/ano, em função dos cenários avaliados. 
COSTA, G.G. et al. Simulação de dados para avaliação econômica do sistema de cria de bovinos de corte em pastagem de Brachiaria brizantha cV. Marandu. PUBVET, Londrina, V. 7, N. 16, Ed. 239, Art. 1580, Agosto, 2013.

Tabela 4. Resultados financeiros e de produção ( $\mathrm{kg}$ bd/ha/ano, kg bd/vaca/ano, Eficiência sistema) dos cenários avaliados.

\begin{tabular}{lccccc}
\hline Resultados & \multicolumn{5}{c}{ Cenários } \\
\hline & \multicolumn{1}{c}{ C1 } & C2 & C3 & C4 & C5 \\
\cline { 2 - 6 } & - & - & - & & \\
Financeiro R\$ & $373.720,3$ & $284.355,9$ & $69.829,5$ & $269.603,2$ & $353.166,7$ \\
kgbd/ha/ano & 17,9 & 27,4 & 42,4 & 48,7 & 53,9 \\
kgbd/vaca/ano & 26,8 & 41,1 & 63,6 & 73,1 & 80,8 \\
Eficiência sistema kg & & & & & \\
7,4 & & 11,4 & 17,7 & 20,3 & 22,5 \\
\hline
\end{tabular}

Nos cenários C3, C4, C5 foi feito estabilização do rebanho para atender as 9000 @/ha. Para C3 foram vendidos, 93\% de vacas falhas em 2016 e 12\% de vacas paridas e $100 \%$ das vacas falhas em 2017. C4 foram vendidos $100 \%$ de vacas falhas a partir de 2013, em 2015, 2016 e 2017 vendidos 6, 51 e 35\% das vacas paridas respectivamente. C5 foram vendidos $100 \%$ vacas falhas, em $201535 \%$ vacas paridas e 5\% novilhas 24 a 36 meses, 2016 vendidos 55\% vacas paridas e $22 \%$ novilhas 24 a 36 meses, 2017 vendidos $50 \%$ vacas paridas.

Os resultados obtidos ao final dos 05 anos para o cenário (C1) demonstrou que a margem liquida do sistema foi de $\mathrm{R} \$-399.434,70$, devido à baixa taxa de natalidade nessa ocasião, resultando em menor número reduzido de bezerros para venda, sendo a venda de vacas vazias apenas $50 \%$ o que não e o suficiente para cobrir os gastos com a mantença do rebanho para a produção.

Com taxa de bezerros desmamados por ha/ano baixa, de $17,9 \mathrm{~kg}$, e o quilograma de bezerros desmamados por vacas expostas a reprodução de 26,8 $\mathrm{kg}$ e eficiência do sistema de 7,4 kg por $100 \mathrm{~kg}$ de vaca exposta, observa se 
que o (C1) de acordo com Anualpec (2011) esta próxima a realidade vivenciada na pecuária de cria atual, mostrando assim que a maioria dos produtores de bezerros atualmente no Brasil está vivendo endividados ou pagando para produzirem.

Foi observado que com o aumento da taxa de natalidade em 10 pontos percentuais passando de 60 para $70 \%$ teve-se aumento na margem liquida de $\mathrm{R} \$ 102.279,30$, mas ficando ainda com margem liquida negativa de $R \$$ $297.155,40$, e aumento de $65 \%$ nos índices de $\mathrm{kg} \mathrm{bd/ha/ano,} \mathrm{kg} \mathrm{bd/vaca/ano}$ e eficiência do sistema.

Obteve-se aumento na receita liquida de $\mathrm{R} \$ 227.325,90$, e $54,70 \%$ de acréscimos encontrados na situação anterior para os índices de $\mathrm{kg}$ bd/ha/ano, $\mathrm{kg}$ bd/vaca/ano e eficiência do sistema, no C3 quando se passa de 70 para $80 \%$ de taxa de natalidade e $10 \%$ a mais na taxa de natalidade das novilhas. Foi feito estabilização do rebanho nos dois últimos anos para atender as 9000 @/ ha, sendo vendidos, 93\% de vacas falhas em 2016 e $12 \%$ de vacas paridas e $100 \%$ das vacas falhas em 2017.

No (C4) foi possível obter maior ganho, aumentando, no entanto, apenas $5 \%$ no percentual de natalidade de vacas e novilhas, na qual a taxa de natalidade chega a $85 \%$ e $65 \%$ respectivamente, passando a ficar positiva sua margem liquida, correspondendo a lucratividade de $\mathrm{R} \$ 272.290,50$ / ano, e aumento nos índices de $\mathrm{kg}$ bd/ha/ano e kg bd/vaca/ano, além de melhoria na eficiência do sistema de 15\%. Conforme Esteio, (2012) este índice de produção pode ser alcançado através do avanço das biotecnologias da reprodução em conjunto com o melhoramento genético, onde esse percentual já e alcançado por fazendas de Goiás.

Já uma situação considerada excelente, na qual já é realidade, pode ser exposta no (C5) com taxa de natalidade de $90 \%$ de vacas e $70 \%$ nas novilhas que emprenham mais cedo aos 18 meses. Nesse cenário foi encontrado lucro de $R \$ 353.166,7 /$ ano, e reduzindo o gasto com animais ociosos, conforme preconizado por Fries (2003) que registra gastos excessivos com animais improdutivos mantidos na propriedade. 
Os valores obtidos de $\mathrm{kg}$ bd/ha/ano, $\mathrm{kg}$ bd/vaca/ano e eficiência do sistema foram $53,9,80,8$ e 22,5 respectivamente, mostrando que o sistema tem desmamado $80,8 \mathrm{~kg}$ de bezerros por vaca e novilha exposta a reprodução. Estes valores não foram maiores devido à capacidade da propriedade onde foi limitada em $9.000 @$, tendo assim que descartar animais o que foi tomado como decisão o descarte de vacas mais velhas mesmo que parida, e conservando novilhas ainda que não estando em estação de monta, sendo o intuito incorporar genética.

A lucratividade de um rebanho mais precoce e de taxa de natalidade maior aqui apresentado foi contrario ao apresentado por Monsalves (2008), o qual reportou maior lucratividade em rebanhos não precoce e com $10 \%$ a menos na taxa de natalidade informando que o rebanho de maior rentabilidade é devido a menor gasto com infra estrutura e alimentação diferenciada para as novilhas precoce, o que levou a uma diferença maior entre receita e despesas, informando ainda que a receita foi maior no tratamento mais precoce porem a despesa também foi muito alta. O que no trabalho apresentado não aparece por ter sistema igual a todos os tratamentos.

Comparando o (C2) que seria a média nacional reportada por Anualpec, (2011) com o cenário que representa realidade possível hoje em Goiás (C4) segundo Esteio (2012), observa-se aumento de $\mathrm{R} \$ 569.445,90$ / ano no lucro do sistema, e aumento em eficiência de $8,9 \mathrm{~kg} / 100 \mathrm{~kg}$ de vaca exposta à reprodução, assim como aumento de $32 \mathrm{~kg}$ bd/vaca/ano.

\section{Conclusão}

O sistema pecuário de cria hoje no Centro-Oeste está bem desenvolvido, porém pouco distribuídos o que mostra ainda termos índices tão baixos de natalidade não passando dos $65 \%$, deixando o produtor no prejuízo.

É possível melhorar o C1 com medidas de manejo, utilizando das biotecnologias disponíveis, mas sempre observando o limite ótimo de produção e lucratividade, o que pode ser observado nos cenários C4 e C5 onde a 
COSTA, G.G. et al. Simulação de dados para avaliação econômica do sistema de cria de bovinos de corte em pastagem de Brachiaria brizantha CV. Marandu. PUBVET, Londrina, V. 7, N. 16, Ed. 239, Art. 1580, Agosto, 2013.

propriedade com estes índices tem lucro de $\mathrm{R} \$ 272.290,50$ e $\mathrm{R} \$ 353.166,7 /$ ano.

\section{Referências Bibliográficas}

BERETTA, VIRGÍNIA; LOBATO, JOSÉ FERNANDO PIVA; MIELITZ NETTO, CARLOS GUILHERME A. Produtividade e Eficiência Biológica de Sistemas Pecuários de Cria Diferindo na Idade das Novilhas ao Primeiro Parto e na Taxa de Natalidade do Rebanho no Rio Grande de Sul. Revista brasileira dezootecnia., 30(4):1278-1286, 2001.

EQUIPE SCOT CONSULTORIA.

HTTP://www.scotconsultoria.com.br/noticias/gestao/21200/pecuaria-sustentavel-e-pecuariaprodutiva.htm, Acesso em: 5 de maio de 2012.

ESTEIO INSEMINAÇÃO PRODUTOS E SERVIÇO. Relatório de campo estação de monta IATF. 2012.

FRIES, LUIS ALBERTO. Genética para um sistema de produção de ciclo curto. I simpósio da carne bovina: da produção ao mercado consumidor, São Borja, 28-30/05/2003, UFRGS/Cooperativa Tritícola SamborjenseLtda

LOBATO, J.F.P. Tecnologias necessárias para a pecuária de corte eficiente e competitiva. In: Ciclo de palestras em produção e manejo de bovinos, 6., Canoas, 2001. Anais... Canoas: ULBRA, 2001. v.1, p.29-48.

MEIRELLES, F. D. P. Modelo computacional de um rebanho bovino de corte virtual utilizando Simulação de Monte Carlo e Redes Neurais Artificiais. 2005. 105f. Tese (Doutorado em Qualidade e Produtividade Animal) - Faculdade de Zootecnia e Tecnologia de Alimentos, Universidade de São Paulo, Pirassununga. 2005.

MONSALVES, FERNANDA MARIA. Valor Econômico e impacto da seleção para precocidade reprodutiva de fêmeas na raça Nelore. Dissertação apresentada à Faculdade de Ciências Agrárias e Veterinárias - 